ITC $1 / 49$

Information Technology and Control

Vol. 49/ No. 1 / 2020

pp. 161-178

DOI /10.5755/j01.itc.49.1.24113
Dynamic Scheduling Algorithm for Delay-Sensitive Vehicular Safety Applications in Cellular Network

Received 2019/08/29

Accepted after revision 2019/11/24

HOW TO CITE: Wu, Q., Fan, X., Wei, W., \& Woźniak, M. (2020). Dynamic Scheduling Algorithm for Delay-Sensitive Vehicular Safety Applications in Cellular Network. Information Technology and Control, 49(1), 161-178. https://doi.org//10.5755/j01.itc.49.1.24113

\title{
Dynamic Scheduling Algorithm for Delay-Sensitive Vehicular Safety Applications in Cellular Network
}

\section{Qiong Wu, Xiumei Fan, Wei Wei}

School of Automatic and Information Engineering, Xi'an University of Technology, China; e-mails: wuqiongysu@126.com,xmfan@xaut.edu.cn,weiwei@xaut.edu.cn

\section{Marcin Woźniak}

Institute of Mathematics, Silesian University of Technology, Poland; e-mail: Marcin.Wozniak@polsl.pl

Corresponding author:xmfan@xaut.edu.cn

The vehicular safety applications disseminate the burst messages during an emergency scenario, but efforts to reduce the delay of communication are hampered by wireless access technology. As conventional VANET (Vehicular ad-hoc network) connected intermittently, the LTE (Long Term Evolution) based framework has been established for the vehicular communication environment. However, resource allocation which is affected by many factors, such as power, PRB (physical resource block), channel quality, are challenging to guarantee the safety services QoS (Quality of Service) in LTE downlink for OFDM (Orthogonal Frequency Division Multiplexing). In order to solve the problem of safety message dissemination in LTE vehicular network, we proposed a delay-aware control policy by leveraging a cross-layer approach to maximize the system throughput. First, we model the resource allocation problem using the queuing theory based on the MISO (multi-input single-output). Second, the method casts the problem of throughput and latency for dynamic communication system into a stochastic network optimization problem, and then makes tradeoffs between them by Lyapunov optimization technique. Finally, we use the improved the branch and bound algorithm to search for the optimal solution in system capacity region for these decomposed subproblems. The simulation results show that our algorithm can guarantee the delay while maximum system throughput.

KEYWORDS: Vehicular network, LTE, Resource allocation, Branch and Bound, Delay control. 


\section{Introduction}

All types of architecture for the vehicular communication networks rely on the wireless access technology, which is usually at the bottom of the communication protocols and provides air interface, in the process of exchanging safety message between mobility vehicle nodes [33], [27]. There are two typical wireless access technology for safety message in vehicular network-DSRC (Dedicated Short-Range Communication) and cellular network [5], [3], [14]. In the previous scholarly output, the research of access technology mostly focused on VANET. It transmits messages by cooperative communication between the vehicle nodes without the participation of the centralized infrastructure. The wireless access technology of VANET is DSRC (Dedicated Short-Range Communication), but it also has some limits.

DSRC is based on architecture of ad-hoc network which has smaller coverage range and connects with other nodes intermittently. There is a lack of deterministic guarantees of service QoS because that the safety message is possible to be dropped when the communication system and links are overloaded or fail [15], [2].

In the vehicular safety application, the types of safety message are divided into two classes. The first is decentralized environment notification message (DENM) which is mainly used in the emergency brake and road alert. The second class is cooperative awareness message (CAM) which is mainly used to transmit the vehicular safety message periodically with $100 \mathrm{~Hz}$, such as vehicular speeds and brake coefficients [23]. However, the lower communication delay and the higher reliability are challenging to realize the broadband access and data sharing of mobile vehicles.

Recently, the researches have had a trend to focus on the cellular technology. In contrast to the DSRC, the coverage areas of Base Station in LTE are larger. The communication links will be established as long as the mobility nodes are in the cell of eNodeB [18]. Indeed, cellular network technology which uses new modulation technique has advantages in many aspects than DSRC, i.e., bandwidth, frequency and transmission rate. For example, LTE can provide a Round Trip Time theoretically lower than $10 \mathrm{~ms}$, and transfer latency in the radio access up to $100 \mathrm{~ms}$ [19]. This is especially beneficial for delay-sensitive vehicle safety applications. The reason mentioned above motivate LTE technology as a promising wireless broadband access technology to support communication under the vehicular network environment.

\subsection{Related Works}

There are many factors that influence the QoS of LTE communication system, i.e., high-order modulation, synchronization, access control strategies, etc. [6], [20], [32]. To achieve the desired system performance and QoS service for the safety message in cellular network, some physical layer technique which impact message dissemination of the cellular-based vehicular network should be considered, such as modulated technique and wireless channel model. Problems of resource allocation and scheduling are also key challenges to be solved in the cellular network [11]. The radio resource management (RRM) algorithms of LTE usually consider three problems - QoS requirement of different users, system capacity and users' fairness. Some studies have made progress in radio resource management of cellular network. Essentially, it can be modeled as an optimization problem for maximizing the utilization of time-frequency resource in the wireless network.

The core work of the problem mentioned above in the vehicular network is downlink scheduling. The vehicle nodes disseminate safety messages over wireless channels under some control strategies containing channel access and power control, where channel access strategies assign the limited radio resource dynamically by sharing the wireless channels and power control techniques allocate the transmission power to compensate the shadow fading and path loss of radio signals. Scheduling is a process which radio resource is allocated to each user in an optimized way. Resource is allocated dynamically to match the user channel time-varying condition and increase the throughput in term of requirement of applications and system resource capacity.

Cellular network has been studied in disseminating the low-latency message. The PHY layer of cellular network is based on the OFDM technique [7], [9]. In [8], the author introduces the basic scheduling algorithms of OFDM mechanism in the application of ve- 
hicular safety service. They are round robin and best CQI (Channel Quality Information) algorithm [1]. The round robin algorithm can get the user fairness performance while the best CQI can get the max system throughput. The PF (proportion fairness) algorithm makes a tradeoff between throughput and user fairness [29], [24]. However, the above studies rarely have focused on the service delay of information and therefore they are not suitable for the vehicular safety message. With higher requirements for reducing the latency of the safety message, the scheduling algorithm for vehicular safety application is also the main research problem. In the case of the higher density of vehicular nodes, many vehicular nodes share the wireless channel and the message burst at the peak of traffic flow, so we need to allocate the limited resource to each user. The system capacity and packet buffer are limited, so it will cause the data buffer congestion or loss of packets if the safety message burst. This problem can cast as the contradictory between the limit frequency resource and the requirement of user QoS services.

The Lyapunov optimization technique, which has been applied to the satellite communication system, is firstly proposed in [22]. It studies the resource allocation problem in multi-hop radio networks by scheduling the active links with backpressure routing and also studies the relationship of capacity achieved region and data transmit rate. In [17], the author plus the penalty function with the Lyapunov drift to extend this method which called Lyapunov optimization technique to study the relationship between the queue stability and user fairness. In recent years, this technique has been applied to the research of control policy for many large communication systems. Paper [12] studies the problem of licensed spectrum sharing with two BSs in the same cell. It uses Lyapunov optimization to solve the spectrum bargaining problem. Paper [13] solves the problem of online rate control and power allocation in the non-orthogonal multiple access system. Paper [20] studies the offload schedule problem of IoT equipment in the edge computing with leveraging the Lyapunov technique, and then solve it by decomposing this problem into the Knapsack problems. Paper [29] presents a lightweight secure self-authenticable transfer protocol for communications between Edge nodes and Fog nodes, which could be also applied in the context of vehicular networks.

\subsection{Main Contribution}

The contradiction between limited resources and user service requirements require efficient resource scheduling solution. We design the resource allocation scheme by cross-layer schedule and access control with using Lyapunov optimization technique, it makes tradeoffs between these factors while disseminating safety messages under LTE communication framework. The main work of this paper con-

Figure 1

Architecture of LTE-based vehicular Network

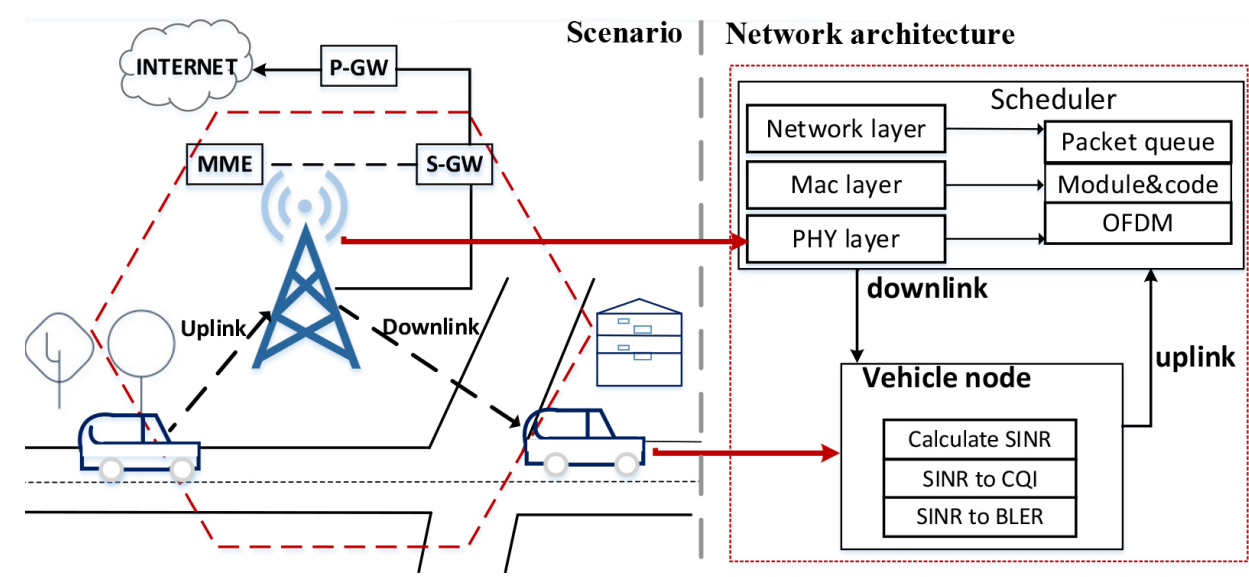


Figure 2

The scheduling flow of LTE-based vehicular Network

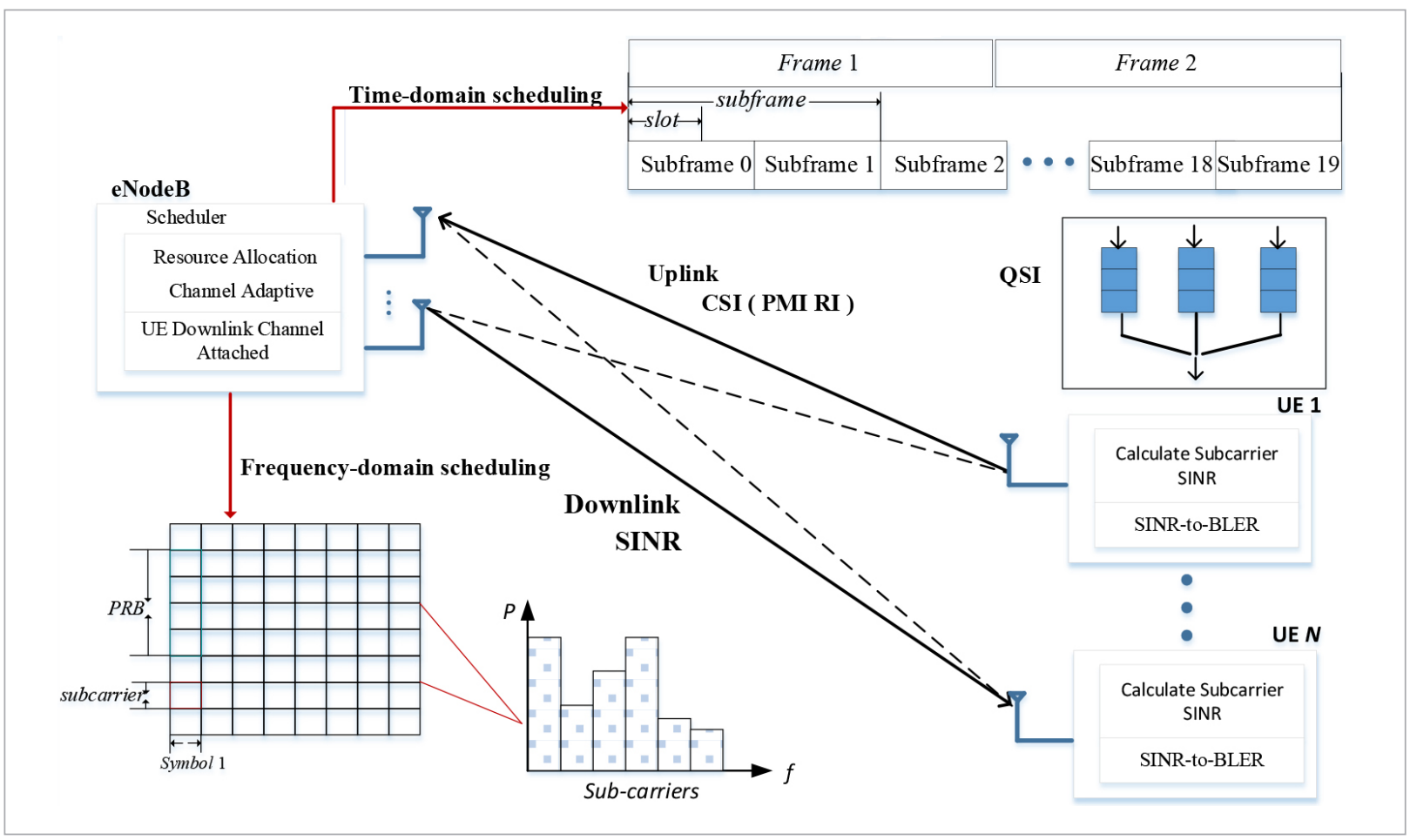

tains three parts: (1) Model and analysis of vehicular communication network. (2) Problem formulation of LTE resource optimization. (3) Get the solutions to the stochastic optimization problem. The method considers the dynamic system stability and delay as a stochastic optimization problem, we get the solution by minimizing the "drift-plus-penalty" of Lyapunov function and then use the classical search algorithm branch and bound to find the optimal solution [13].

The contributions of our proposed scheduling algorithm are mainly concentrated in three points.

- First, we applied the Lyapunov optimization technique for cellular network resource management in tradeoff from two aspects. In one aspect, we use Lyapunov drift plus penalty to tradeoff system stability and system cost which represent the consumption of decision in every slot and use the parameter $\mathrm{V}$ to adjust the weighted between the drift and penalty.

- Secondly, the optimization constraints set are composed by the set of system properties, such as power, the queue backlog. The network controller makes a decision by minimizing drift and penalty and allocates the resource dynamically in every slot.

- Third, because the vehicular node moves fast, the link quantity is also a factor that we consider. With strict and diversified demand for QoS, the traditional hierarchical design is difficult to meet. Therefore, we design a cross-layer schedule policy to improve the reliability and efficiency of safety message by reducing the message delay, and the network resource management mainly concentrates on the physical layer power allocation and network buffer.

The rest of this paper is organized as follows. In Section 2, we introduce the preliminaries about the Lyapunov technique. In Section 3, the communication scenario and formulation scheduling problem are introduced. In Section 4, we study the problem of maximizing the utility of time average throughput, then use Lyapunov optimization framework of the stochastic network to decompose this problem into subproblems to meet delay QoS and system capacity. In Section 5, we present an experimental evaluation. 


\section{Preliminaries}

In this section, we briefly introduce the preliminaries of the Lyapunov technique. It is a challenge to find a solution to trade-off the delay and stability relationship in wireless network. In the first step of designing a cross-layer control policy, we build on system analysis work on stability theory in the sense of Lyapunov. This theory is first proposed by Russian mathematician Lyapunov to describe the stability of the dynamic system in $\varepsilon-\delta$ language. Lyapunov stability theorem is the basis of the Lyapunov analysis framework.

The dynamic system with differential equation $\dot{x}(t)=f(x, t)$ moves between circles with radius $\varepsilon$ and $\delta$ in phase portrait. If the movement trajectory stays near the equilibrium point, so the system is stable. If the movement trajectory is going back to the origin point, it is asymptotic stability, as is shown in Figure 3.

\section{Figure 3}

A schematic of Lyapunov stability in phase portrait

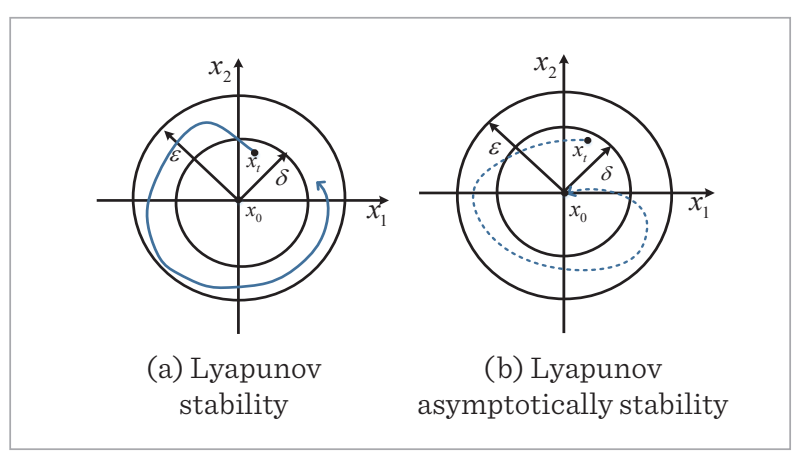

The origin (equilibrium point at the origin) is stable in the sense of Lyapunov or simple stable if

$$
\begin{gathered}
\forall t_{0}, \quad \forall \varepsilon>0, \exists \delta\left(t_{0}, \varepsilon\right): \quad \text { if }\left\|x\left(t_{0}\right)\right\| \leq \delta\left(t_{0}, \varepsilon\right) \\
\Rightarrow t \geq t_{0} \quad\|x(t)\| \leq \varepsilon .
\end{gathered}
$$

The origin is an asymptotically stable equilibrium point if it is stable and in addition:

$$
\begin{aligned}
& \exists \delta\left(t_{0}\right) \geq 0: \quad \text { if }\left\|x\left(t_{0}\right)\right\|<\delta\left(t_{0}\right) \\
& \Rightarrow \quad \lim _{t \rightarrow \infty}\|x(t)\|=0 .
\end{aligned}
$$

This theory does not only define what is the stability but also gives ways to judge whether the complex system is stable. In simple terms, we should find a scalar-valued function, which is called a Lyapunov function to represent the system energy. When the system status deviates from equilibrium, the Lyapunov function increases. The Lyapunov direct method is an efficient method for judging the system stability, we construct the Lyapunov function which is called energy function to represent the dynamic characters. It makes the system not to departure from the stability point by keeping asymptotic steady and general Lyapunov steadily when system subject to external interference.

\section{Problem Formulation}

\subsection{Communication Scenario for LTE Framework}

We consider a simple system-level communication scenario with a single cell and multi-vehicles as illustrated in Figure 1. The cellular-based vehicular network can be divided into two parts: communication scenario and layer architecture.

First, we consider the communication scenario of cellular-based vehicular networks. The communication between the vehicle nodes contains two steps in the framework. In the first phase, the mobility vehicle node transmits the message to the Base Station through the uplink channel. Then in the second phase, the other vehicular nodes which are located in the same cell region receive the message over OFDM downlink channel. The access architecture of the LTE contains access network - EPC and core network - E-UTRAN, so cellular technology has the advantages in link quantity than traditional VANET [25]. The eNodeB connected with the P-GW (packet gateway) and S-GW (service gateway) in E-UTRAN, and the message dissemination between the eNodeB and vehicles use the downlink channel on PDSCH (physical downlink shared channel) in LTE. The vehicular nodes delivery message of vehicle and road by sending the CAM periodically. The dissemination process of safety message must be meet the requirement of delay QoS and ensure the reliability at the same time.

After understanding the framework of the cellular vehicular network, another problem which is needed 
to be elaborated is the communication mechanism of the wireless downlink. In the Architecture of eNodeB, there exist the PHY layer, Network layer, and the function of each layer is packet routing [4], modulation and coding, OFDM, respectively.

The designing of the eNodeB scheduler is the most important task. For the vehicles, the nodes upload the Block Error Ratio (BLER) which is calculated with the received signal quality periodically. The resource allocation is mainly on mapping transmitted data into Physical Resource Block (PRB). In other words, we should allocate the time and frequency domain resource to each vehicular node in the subframe of slot $t$.

\subsection{Problem Statement}

We will introduce every layer of the network architecture in details. As is illustrated in Figure 2, there exists a wild class of network problems to be solved in dissemination of safety message. After understanding the framework and mechanism of the cellular-based vehicular network, we consider the function of dissemination. They can be divided into three subproblems: user utility of performance, network stability, and radio resource allocation in PHY. In our vehicular network communication scenario, the problem can be cast into guaranteeing the message latency of safety applications. In order to solve this problem, we must design an optimization method to solve the three sub-problem mentioned above together under the cross-layer control strategies to schedule system resource dynamically.

The PHY layer and NWK layer were modeled separately which includes the model of the packet buffer in the network layer, the allocation model of a resource power in the frequency domain. In the following, we will describe the network communication process which is in the LTE vehicular network and model problem of dynamic scheduling. We have illustrated the network architecture of vehicular network-based LTE, and then we describe the more details of the system in different communication models.

In order to guarantee that safety message is disseminated, we will use two types of system state vector to solve our problem. Queue length information (QSI) is used to assure the safety message delay, and channel length information (CSI) is used to the maximum utilization rate of wireless spectrum.
First, QSI is represented by $Q_{l}(t)$. Second, the power will be allocated based on the CSI. The purpose of CSI is to feedback the UE channel quantity for eNodeB. The eNodeB choose the suitable coding schematic and modulation rate to transmit the data in PDSCH. The better the link quantity, the more data are to be transmitted in the unit slot. As is shown in Figure 2, process of the message dissemination is divided into two parts: the communication of downlink channel and uplink channel.

First, we describe the wireless channel transmission workflow briefly.

1 In the current subframe, eNodeB creates a list of downlink flows having packets to transmit.

2 The packets queue length and the CQI feedback are stored for each flow. For each flow, the weight is calculated.

3 The flow with the highest weight is given the resource blocks to scheduling. For each flow, eNb computes the transportblock (TB) size, the amount of data to transmit during each TTI.

4 eNodeB uses AMC to map the CSI which contains the (PMI, RI, CQI) feedback with proper modulation and coding scheme.

Second, we introduced the workflow of user scheduling in the time and frequency domain respectively in details which are described in Figure 2. The basic unit of time domain is the frame. It can be divided into ten subframes. The duration of the subframe is $1 \mathrm{~ms}$ which is equal to the minimize scheduling slot - TTI. One subframe contains two slots. In the frequency domain, the basic unit is resource block (RB). Each RB contains twelve sub-carriers which are orthogonality with each other, and its bandwidth is $180 \mathrm{kHz}$. That is to say, if the system bandwidth is $20 \mathrm{Khz}$, it contains a $100 \mathrm{RB}$.

In the MISO system, the design of eNodeB scheduler is the core work for LTE, which allocates the radio resource in an optimal manner to ensure that the communication system can service as many users as possible to maximize system utility. The method we proposed uses network utility as the optimization objection, adopting the mathematical programming method, and finding the optimal solution to design the optimal network scheduling protocol. The details of the system model will be described in the following section. 


\section{Problem Solution}

For the problem mentioned above, we will cast it into a mathematic model by using the different theory in every network layer. The model parameters are provided in Table 1. Then we illustrate the communication problem of LTE-based vehicular network.

Table 1

Notation summary

\begin{tabular}{l|l}
\hline Symbol & \multicolumn{1}{|c}{ Description } \\
\hline $\bar{P}$ & Average power constraint for eNB \\
\hline$p(t)$ & $\begin{array}{l}\text { Transmission power allocated to Node }(\mathrm{m}, 0) \\
\text { in } \mathrm{DL} \\
\text { Over RB k in subframe } \mathrm{t}\end{array}$ \\
\hline$H_{l}$ & $\begin{array}{l}\text { Channel gain of RB j between user l and the } \\
\text { downlink of eNodeB in subframe } \mathrm{t}\end{array}$ \\
\hline$\gamma_{t}$ & $\begin{array}{l}\text { Transmission rate for Node (m, } \mathrm{k}) \text { in } \mathrm{DL} \text { in } \\
\text { subframe } \mathrm{t}\end{array}$ \\
\hline$\alpha_{k}(t)$ & $\begin{array}{l}\text { Admitted amount of data for Node l from the } \\
\text { application layer queue in subframe } \mathrm{t}\end{array}$ \\
\hline$\alpha_{\text {max }}$ & $\begin{array}{l}\text { Upper bound for } \alpha \text { regarding each UE in each } \\
\text { Subframe }\end{array}$ \\
\hline$Q_{l}(t)$ & $\begin{array}{l}\text { Queue length at the eNB for Node (m, } \mathrm{k}) \text { in } \\
\text { subframe } \mathrm{t}\end{array}$ \\
\hline
\end{tabular}

In the wireless communication system, the transmission rate depends on the specific coding scheme. The higher transmission rate requires long block-lengths for coding which also means that there are long message delays.

The optimization goal of the system is to maximize a concave and nondecreasing function of the time-average throughput on each link. Such a function represents a utility function that acts as a measurement of fairness for the throughput vector. For example, the whole system utility will not increase always with the growth of a single variable. This character usually called 'diminish return'. Guaranteeing the delay and reliability of safety message is an optimization problem based on delay and queue stability which is to max the system throughput [16], [10]. Vehicular network access control and scheduling of MAC layer based on delay constraint ensure the stability of the system while meeting the maximum permissible delay of each user constraints, and then there exists a network controller to make the decision when observing the stochastic events occurs. The cross-layer scheduling algorithm has been illustrated in Figure 4.

\section{Figure 4}

The Framework of packet scheduling in a transmitter of eNodeB

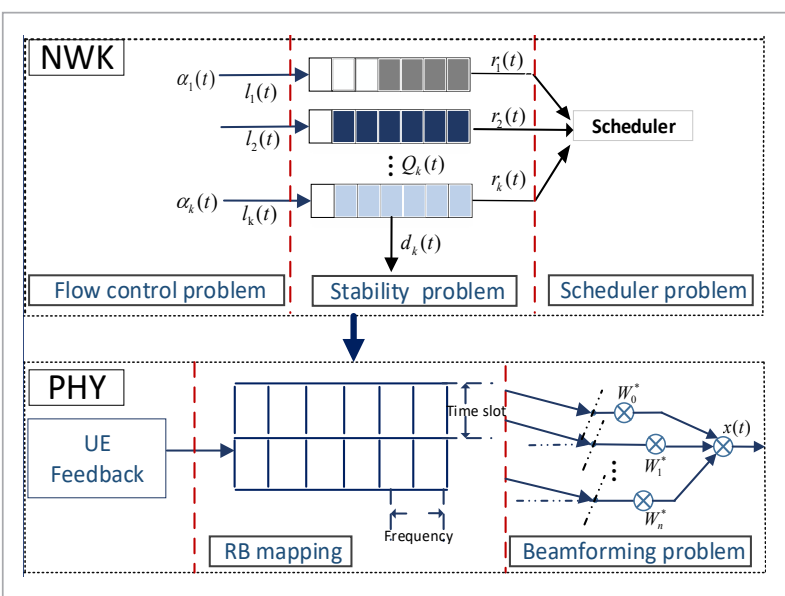

\subsection{Packet Queue Model}

In the Network layer, the main problem is scheduling the resource in the time domain. First, the delay modeling of network queue is set by queuing theory. In every slot $t$, the packet service queue of eNodeB for every vehicle arrives at the network layer according to the certain probability, the packet is cached in the buffer and then access the MAC layer under the control of the scheduler. We assume the network works in the discrete time slot set $t \in\{1,2, \ldots . . . n\},[t, t+\tau)$ is the continuous time interval in slot $t$ and $t+\tau$, the resource allocation and date transmission persist in the consist time $\tau$. The packets are waiting for scheduling according to the FIFS. Arrival process of packets in every traffic flow is i.d.d stochastic process.

In the link $l, l=(1,2, \ldots . k)$, we set the arrival data packet rate of arrival data and service data is $\alpha_{\mathrm{k}}(t)$ and $r_{k}(t), 0 \leq \alpha_{k}(t) \leq A_{\max }$. The queue length in the buffer of the link $l$ is $Q_{k}(t)$ which defined over integer time slot $t=\{1,2, \ldots\}$, represent the contents of a single-server discrete time queueing system. The set $Q=\left[Q_{1}(t), \ldots, Q_{k}(t)\right]$ represents the queue vector of the waiting packet which is also called queue backlog transmitted in the NWK layer. Dynamic evolution equation of queue $Q_{k}(t)$ in link $k$ is given: 


$$
Q_{k}^{t+1}=\left[Q_{k}^{t}-r_{k}^{t}\right]^{+}+a_{k}^{t} .
$$

For all link $k \in L$, where $\alpha_{k}^{t}$ is defined as the number of the packet of arriving at link $l, r_{l}^{t}$ are queue service data of link $l$ in slot $t$. [ ] $]^{+}$represent getting the max value between $Q_{k}^{t}-r_{k}^{t}$ and zero, because the value of queue length is nonnegative. The arriving rate of the data packet obeys the Poisson distribution, $E\left\{\alpha_{l}^{t}\right\}=\lambda_{l}$ is the average data arrival rate.

The two property of the data queue must be considered: queue stability and boundedness of queue backlog. In the sense of packet queue, stability means that the input packet is approximately equal the service rate in a long time. For the entire system this means that the queue can server the packet quickly when the message burst in safety application, e.g. traffic accident broadcast. The boundedness means that the queue capacity is finite and the queue backlog must lower than the boundless to avoid network congestion. Consider the $k$ th-queue of the multiple-access system in Figure 4 and the server model of packet with $\mathrm{M} / \mathrm{M} / 1$ queue is in Figure 5. The arrival time and server time for each packer is stochastic. A packet will be served after the previous arriving packet departure the queue. If the waiting time is too long, the packet should be dropped, as is illustrated with the packet three in Figure 5. As we know the little lemma of queuing theory, the waiting service time of a packet in the queue is equal the division between the number of the packet in the queue and the data service rate, the average delay of the system is following:

$$
\overline{\mathrm{D}}=\sum_{i=1}^{L} \overline{Q_{l}} / \sum_{i=1}^{l} \alpha_{l}
$$

For the link l, the relationship between the average delay, the average queue length is established in (4).

\section{Figure 5}

Dynamic model of Packet service on Queuing Theory

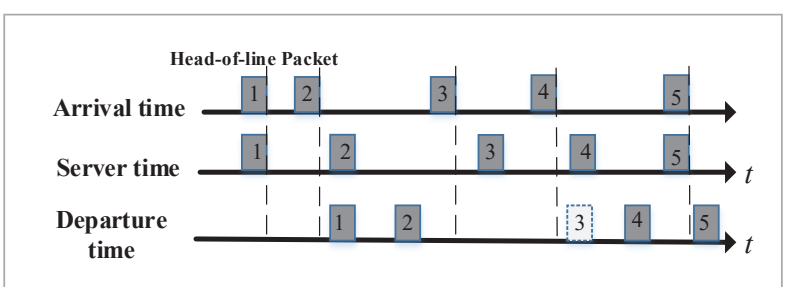

Indicates the average length of the data queue to the link $l$, is defined as the following equation:

$$
\bar{Q}_{l}=\lim _{T \rightarrow \infty} \frac{1}{T} \sum_{\tau=0}^{T-1} E\left(Q_{l}\right)
$$

\subsection{Physical Layer Resource Allocation}

The scheduler of LTE eNodeB allocates the resource block to the different user according to the CQI, which is feedback by the mobile nodes. In LTE downlink channel, the resource allocation is a dynamic process which is in order to improve the resource utilization ratio of the communication system. In the physical layer, the main work is scheduling resource of the frequency domain. We set $P=\left[P_{1}(t), \ldots . P_{n}(t)\right]$ the power allocation vector, so that the scheduling strategy of each link of the wireless network is constrained by the given feasible capacity $\Lambda$.

In the time domain, the main work of scheduler is to allocate the subframe slots to each user by the scheduling algorithm. The smallest unit of system resource is $\mathrm{RE}$ (resource element) which is one symbol and one subcarrier, so the system resource can be divided into the resource grid with 7 symbols and 12 subcarriers which are called RB (resource block). In the frequency domain, the scheduler is to allocate the RBs consisted of the orthogonality channel. As is seen in Figure 5, the bandwidth of $\mathrm{RB}$ is $15 \mathrm{kHz}$ and system bandwidth are $100 \mathrm{MHz}$. The quantity of RBs is 100 in the one-time slot.

There are three type signals in the LTE: control signals, reference signals, and the synchronization signal. More details are illustrated in Figure 6. Then we model the physical layer. The link CSI can be modeled by discrete time Markov process. At each time slot $t$, the network controller selects the transmission rate vector by the constraint condition to represent the flow rate of the traffic on link $l$.

In the wireless communication network, the channel may fade or be lost. To max the network capacity and the stability, we use feedback information of the uplink channel to adjust transmit power of every wireless node.

Downlink SINR under the interference is:

$$
\operatorname{SINR}_{\text {downlink }}(t)=\frac{|H(t)|^{2} p_{i}(t)}{\sigma^{2}+\sum_{l \in L, \notin K}|H(t)|^{2} p_{j}(t)},
$$


Figure 6

Downlink physical channel and Resource block allocation

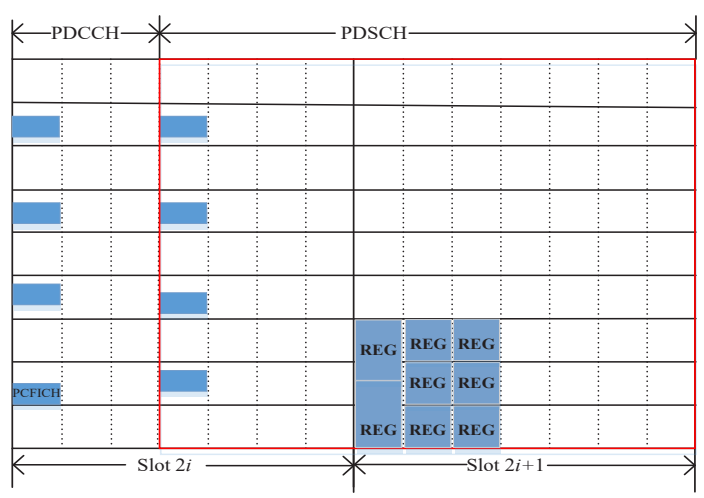

where $\sigma^{2}$ is the noise power. In order to ensure that the signal is received and decoded correctly, the SINR value on the channel cannot be lower than the given threshold.

Then the transmission rate for UE $m$ in the downlink can be expressed as:

$$
r_{m}^{B}(t)=\sum_{j \in J} \log (1+\operatorname{SINR})
$$

We can write the time average transmission rate:

$$
r_{m}^{B}(t)=\sum_{j \in J} \log \left(1+\frac{\left|\mathrm{H}_{i}^{H}\right| p_{i}(t)}{\sigma^{2}+\sum_{m \neq j}\left|H_{m}\right| p_{m}}\right) .
$$

The set of power element $p_{i}(t)$ represents the peak transmit power of the time slot $t$ at the base station for the node $k$; the number of access users in base station is $N, N \leq C$, that each channel $c_{i} \in C$ carries only one node, $P_{k}^{\max }$ is the total power of the system set of power element represents the peak transmit power of the time slot $t$ at the base station for the node $k$; the number of access users in base station is $N$, that each channel carries only one node, $P$ is the total power of the system.

\subsection{System Throughput Problem}

The whole system can be seen as a complex network of the communication queue. We define a set of utility function $\log (1+x)$ representing the satisfaction received by sending data from node $n$ to node $e$ at time average rate of $r$ bits/slot. As is shown in the Figure 7 , the goal is to support a fraction of the traffic demand matrix to achieve long-term throughput that maximizes the sum of user utilities for the whole system through delay control decision and queue backlog decision.

\section{Figure 7}

Communication system and control decision

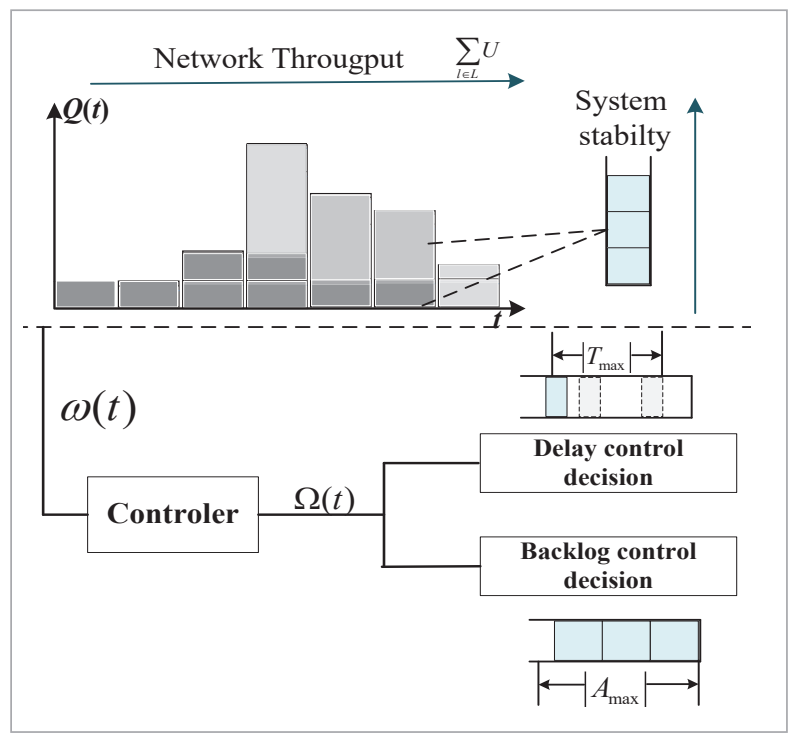

We assume that $\phi_{k}$ is a utility function of maximum throughput. In order to balance between average throughput and fairness, the goal of network control is to maximize the network utility associated with user average throughput. The equation of $\phi_{k}$ is following which represents network fairness:

$$
\phi_{k}=\sum_{k=K s} \log \left[1+\left(\overline{\alpha_{k}}\right)\right]
$$

The utility functions are assumed to be non-decreasing and concave. $\overline{\alpha_{k}}$ is the long average packet arrival rate. This is illustrated in the following equation:

$$
\overline{\alpha_{k}}=\lim _{t \rightarrow \infty} \sum_{k} \frac{1}{\mathrm{~T}} E\left\{\alpha_{k}(t)\right\} .
$$

The above equation represents the optimization objective function for scheduling problem. The problem we faced is making a tradeoff between different factors, such as power, channel, which in order to achieve the desired system outcomes. The essence of making tradeoff in the best way is optimization problem. 
To design a control policy by Lyapunov analysis framework with cross-layer optimization, we will adopt the following four steps in the next section:

1 Model the original optimization problem.

2 Because the problem contains the time average function, auxiliary variable and virtual queue technology is used to transform it into the problem which only obtained the time average variable, then use the Lyapunov optimization framework to analyse this problem.

3 Minimize the Lyapunov drift and penalty function.

4 Analysis of the performance of the proposed algorithm.

In the first step, we set $U$ as the average throughput of node $k$, and it is a continuous strictly convex function which contains the time average variable. We set the delay-aware resource control strategies as the optimization constraint of the scheduler. The above problem can be stated as the network utility model of following optimization problem.

$$
\begin{aligned}
& \text { Maximize } \sum_{k \in K} U_{k}\left(\overline{\alpha_{k}}\right) \\
& \text { s.t } \quad \overline{r_{k}} \geq \overline{\alpha_{k}} \quad \forall k \in K \\
& r_{k}(t)= \sum_{j \in J} \log \left(1+\frac{\left|H_{i}\right| p_{i}(t)}{\sigma^{2}+\sum_{k \in K, k \notin l}\left|H_{K}\right| p_{k}(t)}\right) \\
& \sum_{i=0}^{n} p_{i} \leq P_{k}^{\max } .
\end{aligned}
$$

The above formula is an optimization problem with multi-variables. The core idea of this stochastic optimization problem is how to tradeoff between the queue stability and the system utility to achieve the maximum packet throughput. The next problem is how to search for the solution to this optimization problem. Next section mainly discusses the control and scheduling algorithm based on Lyapunov optimization theory and analysis framework. We have transformed the problem into tradeoff between queue stability and optimization target function.

\subsection{Cross-Layer Resource Scheduling of Lyapunov Optimization}

Because of the time-varying characteristics of the wireless channel, the packet arrival in the network has random characteristics, so the problem belongs to a stochastic network optimization, which can make the cross-layer resource scheduling decision after observing the random sequence. We mainly explore the relation between system stability and delay. For a communication system, the stability means no dropped packets. When the packet is pushed into the queue, the backlog is increasing, the delay will also increase, and the system will be congestion. The stochastic optimization problem is transformed into the stability of the queue by the optimization problem of the time-averaged variable, and the optimization problem is solved by the Lyapunov optimization of drift plus penalty.

\subsubsection{Lyapunov Analysis Framework}

As a stability criterion of a dynamic queue system, the Lyapunov function is a quadratic function, and it presents the system energy, which is constructed with the algorithm of the sum-of-squares. When this function reduced, the system will go to stability. The Lyapunov optimization technique is an application of Lyapunov stability theorem to the communication and queue system, which can achieve the network utility objection while keeping system stability and achieve the performance-delay tradeoff. To deal with the dynamic network state, the Lyapunov optimization technique was widely used for maximizing the network utility. In resource allocation schemes based Lyapunov optimization, iterative search algorithms were widely used to obtain the optimal resource allocation solution. In the following section, we review and introduce briefly the framework based on the stability in the sense of Lyapunov.

In Section 3, we model the LTE communication system by network layer model with its function separately. In this section, we describe the system as a whole in the aspect of the system control to solve the online scheduling problem of data packets. As a stability criterion of a dynamic queue system, the Lyapunov function is a quadratic function, and it presents the system energy which is constructed with the algorithm of the sum-of-squares. When this function is reduced, the system will go to stability. The Lyapunov optimization technique is an application of Lyapunov stability theorem to communication and queue system, which can achieve the network utility objection while keeping system stability and achieve the performance-delay tradeoff. To deal with the dy- 
namic network state, the Lyapunov optimization technique was widely used for maximizing the network utility. In resource allocation schemes based Lyapunov optimization, iterative search algorithms were widely used to obtain the optimal resource allocation solution.

\subsubsection{Queue and System Stability}

In this section, we will solve the resource allocation problem through Lyapunov analysis framework mentioned above. The core idea of scheduling algorithm which solves the cross-layer optimal scheduling algorithm is to use Lyapunov optimization theory to allocate resource dynamically under the constraint that the data queues and virtual queues of the system are stability. Through the queuing theory, we model the problem as designing scheduler of a dynamic system. The study of dynamics originated from the control theory, and the basic foundation is the stability in the sense of Lyapunov. It is also called the Lyapunov directed method. Control technique of this communication queue system is the main problem. We regard the system as a stochastic system, the solution of the optimization is to the stability point of a system which balances the queue stability and delay. We can assume that there exists a network controller in a wireless network by using Lyapunov optimization technology. The input of the controller is queue state information and control state information of wireless network, such as the queue length, channel link, etc.

First, we give the analysis framework of Lyapunov optimization technology briefly. The framework can be divided into three parts: (1) The three technology in the Lyapunov framework: queue stability theory, virtual queue technique and 'drift-plus-penalty' function. (2) Construct the Lyapunov function which is the sum of the square of the virtual queue and data queue and Minimum the function to make the system stability. (3) The Lyapunov inequation. We can find the boundness of minimizing the right-hand-side of the equation to find the optimization solution. How this technique works when it is applied to the design of the cross-layer protocol in the LTE communication system.

Lyapunov drift is a powerful technique for optimizing time averages in stochastic queueing network subject to stability. Lyapunov queue stability is defined as follows:

\section{Lemma 1: if}

$\lim _{t \rightarrow \infty} \sup E\{Q(\tau)\}<\infty$.

The queue is average rate stability, the queue has an upper bounder of time average accumulation, and the queue is strongly stable.

$$
\bar{Q}_{l}(t) \triangleq \lim _{t \rightarrow \infty} \sup \frac{1}{t} \sum_{\tau=0}^{t-1} E\left\{Q_{l}(\tau)\right\}<\infty, l \in L .
$$

If all the queue of the system is strongly stable, the system is called strong stability. The serve and arrival of the queues are i.i.d process.

\subsubsection{Jointly Cross-Layer Scheduling Scheme}

We use the Lyapunov optimization to enforce the queue stability to minimize the drift and penalty of Lyapunov function. Build the set of packet queue and virtual queue, the Lyapunov function is the sum of squares for packet queue and virtual queue:

$$
\Theta(t)=\left\{Q_{k}(t)\right\} .
$$

We define the quadratic Lyapunov function as following and it prepares the degree of system congestion:

$$
L(\Theta(t))=\frac{1}{2} \sum_{k} E\left[Q_{k}^{2}(t)\right] .
$$

First, we use Equation (15) to compute the boundedness on the change in Lyapunov function with Lyapunov drift at $t$ slot and $t+1$ slot:

$$
\Delta_{\text {one-slot }}(\Theta)=L(\Theta(t+1))-L(\Theta(t)) .
$$

The penalty function is the penalty weight $\mathrm{V}$ plus the max throughput utility function:

$$
\Delta(L(t))-V^{*} F .
$$

The most significant feature of this algorithm is that it does not need to know the probability of a stochastic event. After observing, it seeks to minimize a (possibly non-linear, non-convex, and discontinuous) function of control decision.

Next, we solve the upper bound of Lyapunov drift and penalty and decompose this problem into sub-problems and provided an optimal solution for each, then 
we can obtain the control policy solution dependently:

$$
\begin{aligned}
\Delta(L(t))-V^{*} F= & \frac{1}{2}\left[E\left(\sum_{i=1}^{N}\left(Q_{l}^{2}(t+1)-\mathrm{Q}_{l}{ }^{2}(t)\right)\right]-V\left[\sum_{k \in K}\left(\phi_{k}\left(\mathrm{a}_{k}(t)\right)\right]\right.\right. \\
= & \frac{1}{2}\left[\sum_{i=1}^{N}\left(Q_{k}-r_{k}(t)\right)^{+}+\alpha_{k}(t)\right]^{2}-V^{*} F \\
\leq & \frac{1}{2}\left[+\left(r_{k}(t)\right)^{2}-2 \sum_{i=1}^{N} Q_{k}\left(r_{k}(t)-\varepsilon\right)+\right. \\
& \left.+\alpha_{k}(t)^{2}\right)-V\left[\sum_{k \in K}\left(\overline{\phi_{k}\left(\mathrm{a}_{k}(t)\right.}\right)\right] \\
& =M-V E\left\{\sum_{k=1}^{K} \phi_{k}\left(\eta_{k}(t)\right) \mid L(t)\right\}- \\
& \sum_{k=1}^{K} Q_{k}(t) E\left\{r_{k}-a_{k}(t) \mid L(t)\right\} .
\end{aligned}
$$

$\mathrm{M}$ is a non-negative constant, defined as follows:

$$
M=\frac{1}{2} \sum_{k=1}^{K} E\left\{a^{2}+b^{2} \mid L(t)\right\} .
$$

For the first part of the joint optimization problem, we consider the linear programming problem on end to end rate control with the parameter which is the arrival packet every slot in the packet queue:

\section{P1:}

$$
\begin{array}{ll}
\min & -2 Q(t) a(t)-V^{*} \log (1+a(t)) \\
\text { s.t } & 0 \leq a(t) \leq A^{\max } .
\end{array}
$$

The second order deviation of the objection function of $\mathrm{P} 1$, so it is a convex function. We can get the global optimal value of the objection through getting the first order deviation, set the solution of the objection function as following:

\section{Figure 8}

Weighted sum rate problem illustration

$$
a(t)=V / q(t)
$$

The second subproblem is the maximization of the weighted sum rate in the stability capacity region. The weighted sum rate is affected not only by the power, and also by the weighted value which is the queue in the network layer (Figure 8). This solution approach to this sub-optimization problem will be in the next section.

\subsubsection{Power Allocation Problem}

It is an NP-hard problem and also a cross-layer scheduling approach for transmission rate adaptively to maximize the network capacity. The difficulty is how to solve this optimization problem. This problem is the maximum weighted sum rate (WSRmax), which is NP-hard and non-convex [30]. Considering $k$ users and one Base Station, the downlinks cannot work simultaneously owing to the mutual interference of wireless channel. Under the link scheduling strategic, the sum rate of all users achieve the maximum value to improve system throughput.

Here we use the brand and bound algorithm improved by SOCP mentioned in [24] to solve this problem. We will illustrate the WSRmax problem in details on the following three aspects:

1 The description of WSR problem in downlink scheduling.

2 Theoretical analysis and theorem proof.

3 The pseudo code and simulation result.

Assume that $\sigma^{2}$ is the noise power, $Q_{k}(t)$ is the packet actual queue. The balance of delay and packet will affect the system user sum rate. To solve this problem, we rewrite the Eq. (11) as follows:

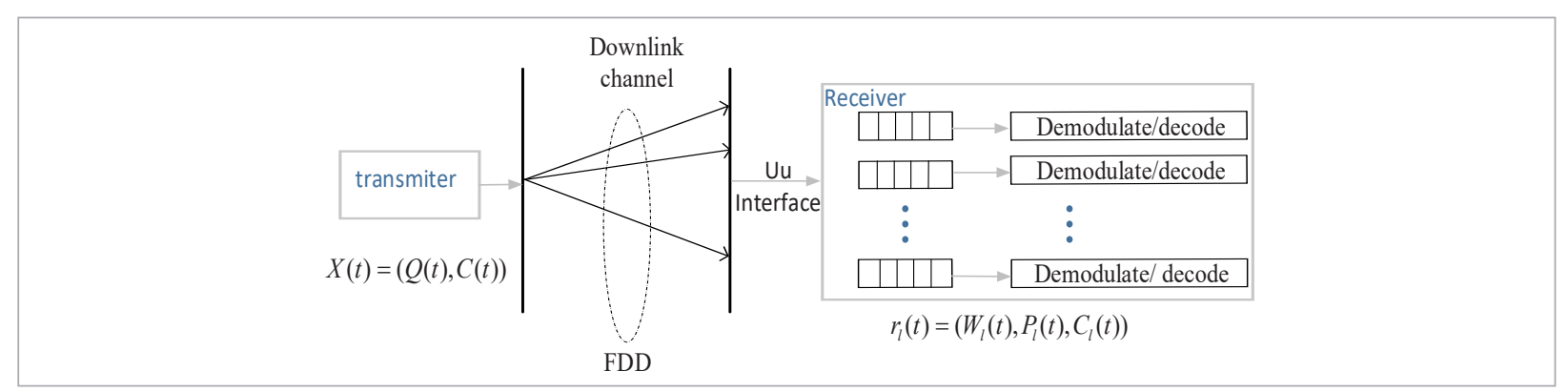




\section{Figure 9}

Capacity throughput region and user rates

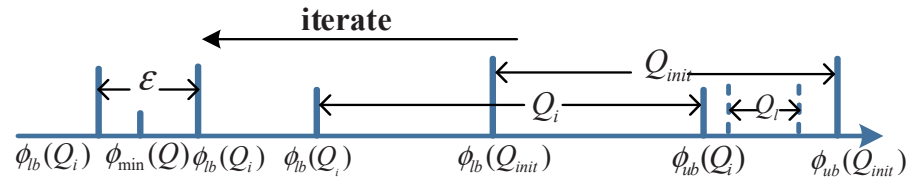

(a) scale of the lower and upper bound

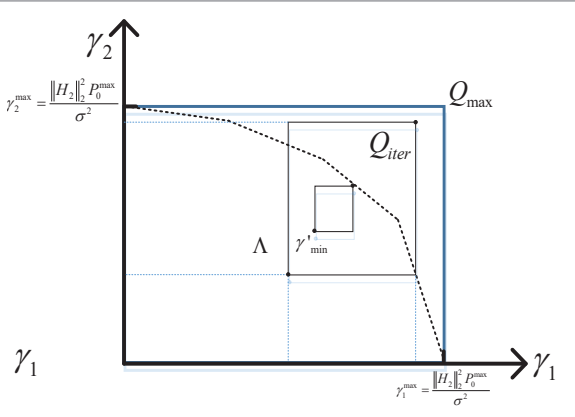

(b) bound the region

$$
\begin{array}{ll}
\min & -\sum_{k \in K}\left(Q_{k}(t)\right) \log (1+\gamma) \\
\text { s.t } \quad & \left\{\Lambda \mid \gamma_{l} \leq 1+\frac{\left|g_{l l} p_{l}\right|^{2}}{\sigma^{2}+\sum_{j \in L, j \neq l}\left|h_{j l}^{H} m_{j}\right|^{2}}\right\} \quad l \in L \\
& \sum_{i=0}^{n} p_{i} \leq P_{k}^{\max } \\
& R_{i} \geq 0 .
\end{array}
$$

Equation (22) is a nonconvex function. This optimization is illustrated by the Figure 9 . The variables are $p_{l}$ and $\gamma_{l}$, we can see that the fading of each sub-carrier is not different, which leads to allocating the different power to max the throughput under the different weights. Weighted coefficients are associated with the backlog in the network layer. It must also consider the interference and coupling among the different wireless links in the MISO system. We use the SOCP (second-order cone optimization problem) and branch-and-bound distributed approach to solve this NP-hard problem. The process can be concluded in the following three steps:

1 Transmit the feasible sets of the original problem into rectangle regions.

2 Prove that the optimal solution of the transformer problem is equal to the origin problem.

3 Use the branch and bound algorithm and second-order cone optimization to search the optimal solution of the transformer problem

First, we set $f_{0}(\gamma)=-Q(t) \log (1+\gamma)$. We relax the feasible set $G$ to $\mathrm{m}$-dimensional rectangle $Q$ under the condition without the interference of other links to simplify the system channel model. The constraint of above problem constructs the new feasible set $\gamma=\left(\gamma_{1}, \gamma_{2}, \ldots . \gamma_{L}\right)$ :

$$
Q_{\text {init }}=\left\{\Lambda \mid 0 \leq \gamma_{l} \leq \frac{\left\|g_{l l}\right\| p_{\text {tran }(l)}^{\max }}{\delta^{2}}, l \in L\right\} .
$$

Second, we set a tighter lower and upper bound for the branch and bound algorithm. The process of the branch and bound is an iterative search, and we illustrate this process using Figure 9(a). We take a two-dimensional sum rate as examples; the capacity region is illustrated as Figure 9(b). We use the branch-andbound algorithm to compute the optimal value iteratively. Each time, we search the upper value and lower value through the function $\Phi_{\text {upper }}(Q)$ and $\Phi_{\text {lower }}(Q)$. The core idea of the branch and bound algorithm which is used to solve the WSRmax problem is to search the feasible set in the rectangle region by iterate splitting the rectangle into the small ones. The node $C$ is called uptoia point and represents the maximized system throughput under the non-constraint condition. It is also called uptoia point. The boundary of the feasible set of optimize problem (22) is called Pareto optimal solution.

$$
\Phi_{l b}^{\text {basic }}(Q)=\left\{\begin{array}{l}
f_{0}\left(\gamma_{\min }\right) \quad \gamma_{\min } \in \Lambda \\
0 \quad \text { otherwise }
\end{array}\right.
$$

This problem can be cast into an optimization problem that finds the solution of $P$ in the feasible set $G$, 
and then transform it to a second-order cone optimization to solve it. The transform process is defined in Equation (25).

We transform the constraint of formulation (22) into second-order conic optimization:

$$
\begin{array}{ll}
\text { find } & p_{1}, p_{2}, \ldots . . p_{L} \\
\text { subject to } & {\left[\sqrt{\left.\square 1+\frac{1}{\gamma_{l}}\right)} p_{l}^{H} h_{l}\right] \geq\left\|\begin{array}{l}
h_{i} P \|, \quad \forall l \in L \\
\sigma
\end{array}\right\|,} \\
& \sqrt{p_{n}^{\max }} \geq\left[p_{n}\right]^{T}, \forall l \in L .
\end{array}
$$

The main idea of the improved BB algorithm is to tighten the upper and lower bounds of each rectangular region by making the rectangular boundary point close to the feasible solution region $\mathrm{G}$, thereby reducing the optimal solution search range and improving the convergence speed of the algorithm. The BB algorithm for WSRmax problem was summarized in following pseudo-code:

Algorithm 1. The branch and bound algorithm for maximizing the weighted sum rate problem

\section{Initialization.}

1: Set $\phi_{l b}^{\text {basic }}\left(Q_{\text {init }}\right)=f_{0}\left(\gamma_{\max }\right) ; \phi_{u b}^{\text {basic }}\left(Q_{\text {init }}\right)=f_{0}\left(\gamma_{\min }\right)$.

Iteration indices $\mathrm{i}=0$ and $\mathrm{k}=0$.

Given the feasible set of $\gamma_{i}$ :

$Q_{i n i t}=\left\{\Upsilon \mid 0<\gamma_{i}<\frac{\left|h_{i j} p\right|^{2}}{\delta^{2}+\sum_{i \notin j}\left|h_{i j} p\right|^{2}}\right\} \quad i=1,2, \ldots l$

BB algorithm iteration:

\section{For slot=1 to $T$}

3 For $i=1$ to $N$

4 Split $Q_{\text {init }}$ into $Q_{1}$ and $Q_{2}$

$5 \mathrm{~L} 1=\min _{\{\mathrm{Q}\}}\left\{\phi_{l b}\left(Q_{1}\right), \phi_{l b}\left(Q_{2}\right)\right\}$

$\mathrm{U} 1=\min _{\{\mathrm{Q}\}}\left\{\phi_{u b}\left(Q_{1}\right), \phi_{u b}\left(Q_{2}\right)\right\}$

6 Pick $Q_{i}$ which contains the minimize $\phi_{l b}\left(Q_{i}\right)$, then split it along the longest side with the two new small rectangles $Q_{i+1}, Q_{i+2}$.

7: update

$\mathrm{L} i=\min \left\{\phi_{l b}\left(Q_{1}\right), \ldots . . \phi_{l b}\left(Q_{i+1}\right), \phi_{l b}\left(Q_{i+1}\right)\right\} \backslash\left\{\phi_{l b}\left(Q_{i}\right)\right\}$

8 update

$\mathrm{U} i=\min \left\{\phi_{u b}\left(Q_{1}\right), \ldots . . \phi_{u b}\left(Q_{i+1}\right), \phi_{u b}\left(Q_{i+1}\right)\right\} \backslash\left\{\phi_{u b}\left(Q_{i}\right)\right\}$
9 Pick the $Q_{i+1}, Q_{i+2}$

10 if $\Upsilon_{\min } \in G$

11 set $\mathrm{V}=\left(\Upsilon_{\min }+\Upsilon_{1, \text { max }}\right) / 2$

12 If $\mathrm{V}$ inside $\mathrm{G}$

$13 \Upsilon_{\text {min }}=$ V else $\Upsilon_{1, \max }=V$

14: if $\left\|\gamma_{1, \max }-\gamma_{\min }\right\| \leq \varepsilon$, the iteration stop else go to step 11 to find the point

15: update the with a new rectangle

16: stopping criterion if the stopping criterion is satisfied, go to step 3 . Otherwise set $i=i+1, k=k+1$.

\section{Results}

The simulation result and analysis should verify the following three sub-problems: (1) What the relationship between V and the queue backlogs is? (2) How optimization performance value changes versus the different V values; (3) What the tradeoff between Lyapunov drift and penalty function is? Figure 10 shows that the change of the relationship between the queue backlog, service rate and arrival rate when the $\mathrm{V}$ is equal to the 50 . With the time-varying characteristics of wireless channel, the user service rate changes with channel state. The weight associated with each user is set up by queue backlog at the current time. At the beginning of the iteration, the arrival

\section{Figure 10}

Capacity throughput region and user rates

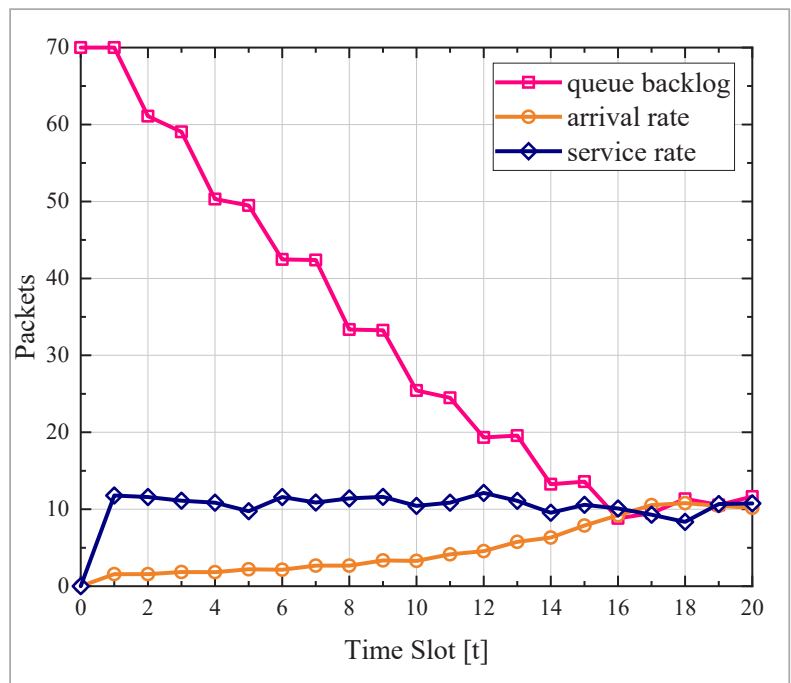


rate is smaller than the service rate. That is because the queue backlog is large, so control policy increases the weights of the service rate, and the arrival rate is small. With the increase of iteration, queue backlog had been reducing, the curve of arrival rate growth to increase the system throughput.

To evaluate the performance of proposed Algorithm 2 , we consider a single fading realization. Figure 11 shows the service rate values of the problem (25) under the control parameters $\mathrm{V}$ is equal to 50,80 and 100. In Figure 11, we can conclude that there are two factors that influence the values of user service rate, channel state and queue weights. When queue backlog is large, weights are also large, so under the same channel state, service rate will be increase.

Figure 11

Weighted rate versus different V

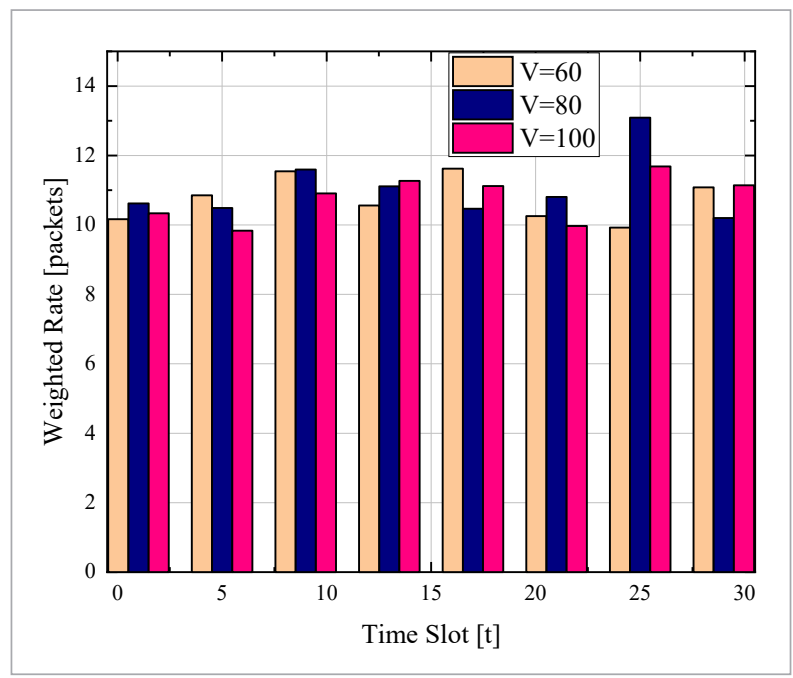

In Lyapunov optimization, the control parameter $\mathrm{V}$ is used to adjust the tradeoff between the Lyapunov drift and optimization objection. In Figure 12, we analyze the fitting curve trend of the arrival rate versus V. The throughput is the sum number of arrived packets of the whole wireless communication system, it represents the system service capability. The arrival rate convergent by the $\mathrm{V} / \mathrm{q}$, so the curve slop is flatter with the increase of $\mathrm{V}$. By contrast with the backlog, the throughput curve is increased with the growth of the control parameter $\mathrm{V}$ with $\mathrm{O}(\mathrm{V})$. We analyze the trend of fitting curve of queue backlog versus different control parameter value in Figure 13. The queue
Figure 12

Arrival rate versus control parameter V

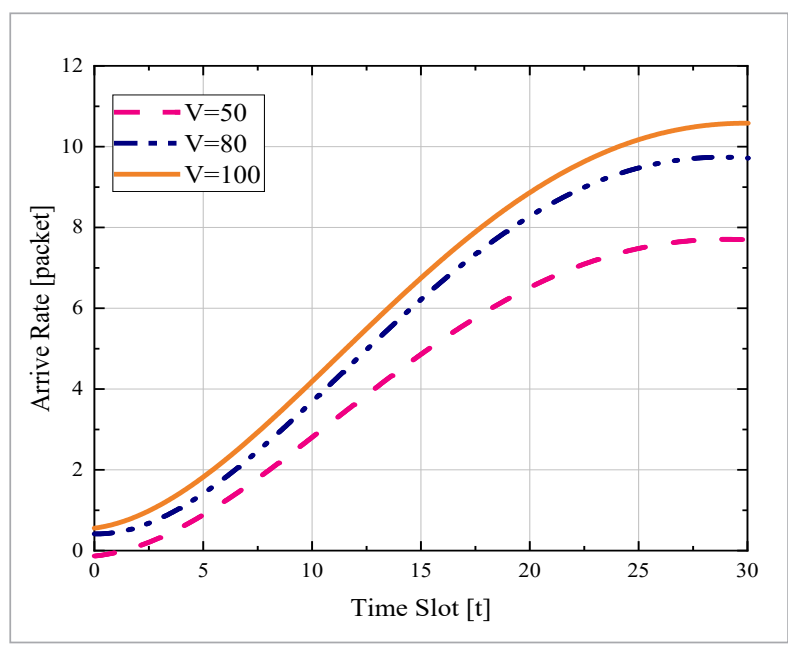

Figure 13

Queue backlog versus control parameters V

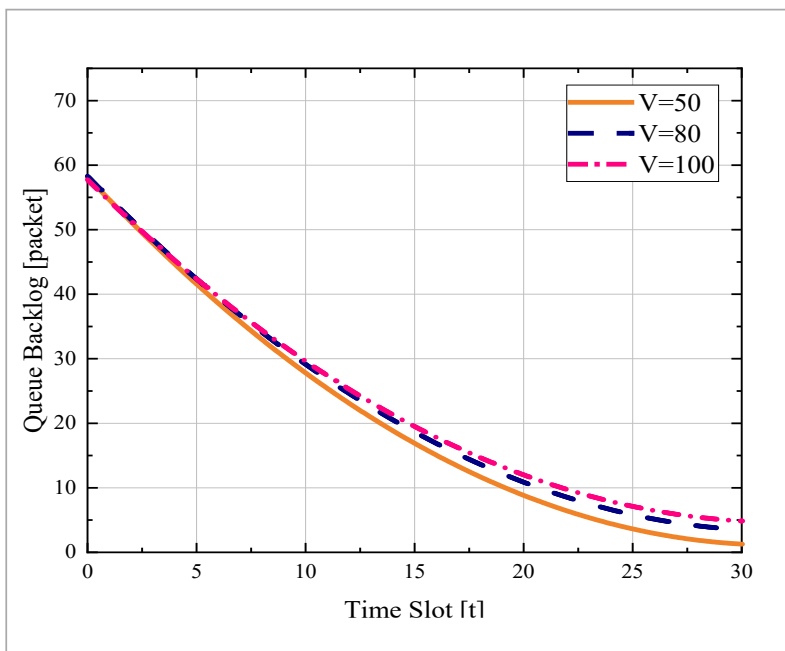

backlog curve decreased with the iteration slot. That is because with the jointing optimization control of arrival rate and service rate, the packets in the buffer will be decreased. In the Lyapunov drift and penalty, the penalty is representing the system throughput. If the value of parameter $\mathrm{V}$ increases, the proportional of the Lyapunov drift decreases, maximum throughput becomes a priority to be considered.

In Figure 14, we analyze the change of buffer capacity versus different SNR. The packet backlog decreases with the iterations, and tend to stable finally un- 
Figure 14

The packet buffer backlog versus SNR

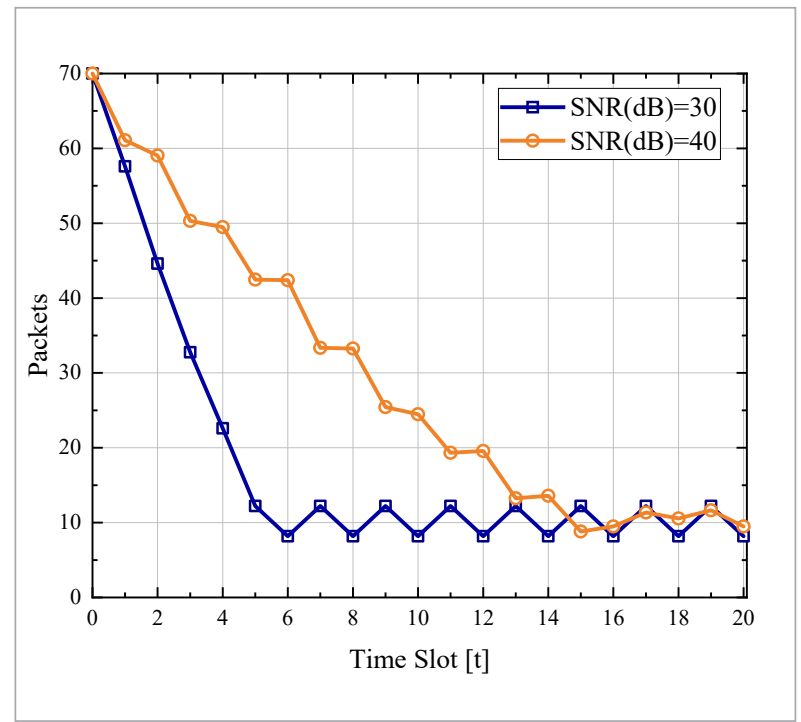

der the queue control policy. With the different SNR value, the decrease slope is different. When the SNR value is equal $40 \mathrm{~dB}$, the slope is flatter. With the decrease of SNR, the packet backlog decreases from 70 to 10 after six times iteration. That is because that the higher SNR denote the better channel state, and the service rate will be large, so the backlog will not congest in the queue buffer.

\section{References}

1. 3rd Generation Partnership Project, «3GPP TS 36.213 version 10.6.0: Physical Layer Procedures». http://www.3gpp.org.

2. 3rd Generation Partnership Project. «3GPP TS 36.211 V10.4.0. Evolved Universal Terrestrial Radio Access (E-UTRA): Physical Channels and Modulation». http:// www.3gpp.org.

3. Abboud, K., Omar, H. A., Zhuang, W. Interworking of DSRC and Cellular Network Technologies for V2X Communications: A Survey. IEEE Transactions on Vehicular Technology, 2013, 65 (12), 9457-9470. https:// doi.org/10.1109/TVT.2016.2591558

4. Alfa, A., Misra, S., Adewumi, A., Salami, F. O., Maskeliunas, R., Damasevicius, R. Implementation of MANETs

\section{Conclusions}

In this paper, we study the problem of safety message dissemination in the downlink of the LTE-based vehicular network. We design a delay-aware control policy under a cross-layer approach to realize the multi-user scheduling and maximize the system throughput based on the MISO system model. In the Lyapunov analysis framework, we decompose the optimization problem into some subproblems such as flow control, delay, transmission rate and power. We obtain the corresponding control policy by solving these subproblems. The power allocation subproblem is weighted sum rate problem and also is an NP-hard. We use the improved Branch-Bound algorithm to search the optimal solution in the feasible set by improving the upper and lower boundaries. In the end, we use MATLAB to simulate our algorithm, and the simulation result satisfied the theoretical derivation.

\section{Acknowledgements}

This work was supported in part by the National Natural Science Foundation of China under Grant 61272509, and in part by the key research and development plan of Shaanxi province under Grant 2017ZDCXL-GY-05-01, and in part by Shaanxi Province Hundred Talent program. This job is also supported by the Key Research and Development Program of Shaanxi Province (No.2018ZDXM-GY-036).

Routing Protocols in WLANs Environment: Issues and Prospects. International Conference on Information Technology Science (MOSITS 2017), Moscow, Russia, December 1-3, 2017, 252-260. https://doi. org/10.1007/978-3-319-74980-8_24

5. Araniti, G., Campolo, C., Condoluci, M., Iera, A., Molinaro, A. LTE for Vehicular Networking: A Survey. IEEE Communication Magazine, 2013, 51(5), 148-15\%.https:// doi.org/10.1109/MCOM.2013.6515060

6. Bao, W., Chen, H., Li, Y. Joint Rate Control and Power Allocation for Non-Orthogonal Multiple Access Systems. IEEE Journal on Selected Areas in Communications, 2017, 25 (12), 2798-2811. https://doi.org/10.1109/ JSAC.2017.2726357

7. Capozzi, F., Piro, G., Grieco, L. A. Downlink Packet Sche- 
duling in LTE Cellular Networks: Key Design Issues and a Survey. IEEE Communications Surveys and Tutorials, 2013, 15 (2), 678-700. https://doi.org/10.1109/ SURV.2012.060912.00100

8. Cui, Y., Lau, V. K. N., Wang, R. A Survey on Delay-Aware Resource Control for Wireless Systems-Large Deviation Theory, Stochastic Lyapunov Drift, and Distributed Stochastic Learning. IEEE Transactions on Information Theory, 2012, 58(3), 1677-1701. https://doi. org/10.1109/TIT.2011.2178150

9. Elsaadany, M., Ali, A., Hamouda, W. Cellular LTE-A Technologies for the Future Internet-of-Things: Physical Layer Features and Challenges. IEEE Communications Surveys and Tutorials, 2017, 19(4), 2544-2572. https://doi.org/10.1109/COMST.2017.2728013

10. Gabryel, M., Wozniak, M., Damasevicius, R. An Application of Differential Evolution to Positioning Queueing Systems. In Artificial Intelligence and Soft Computing (ICAISC 2015), Zakopane, Poland, June 14-18, 2015, 379-390. https://doi.org/10.1007/978-3-31919369-4_34

11. Goldsmith, A. Wireless Communications. Cambridge University Press, Cambridge, 2005.

12. Joshi, S. K., Manosha, K. B. S., Codreanu, M. Dynamic Inter-Operator Spectrum Sharing via Lyapunov Optimization. IEEE Transactions on Wireless Communications, 2017, 16(10), 6365-6381. https://doi. org/10.1109/TWC.2017.2722999

13. Joshi, S., Weeraddana, P. C., Codreanu, M. Weighted Sum-Rate Maximization for MISO Downlink Cellular Networks via Branch and Bound. Signals, Systems and Computers, 2012, 4(10), 2090-2095. https://doi. org/10.1109/TSP.2011.2182346

14. Ju, H., Liang, B., Li, J. Dynamic Joint Resource Optimization for LTE-Advanced Relay Networks. IEEE Transactions on Wireless Communications, 2013, 12(11), 5668-5678. https://doi.org/10.1109/ TWC.2013.092513.121922

15. Karagiannis, G., Altintas, O., Ekici, E. Vehicular Networking: A Survey and Tutorial on Requirements, Architectures, Challenges, Standards and Solutions. IEEE Communications Surveys and Tutorials, 2011, 13(4), 584-616. https://doi.org/10.1109/ SURV.2011.061411.00019

16. Kempa, W. M., Wozniak, M., Nowicki, R. K., Gabryel, M., Damasevicius, R. Transient Solution for Queueing Delay Distribution in the GI/M/1/K-type Mode with „Queued“ Waking up and Balking. International Con- ference on Artificial Intelligence and Soft Computing (ICAISC), Zakopane,Poland, 2016, 340-351. https://doi. org/10.1007/978-3-319-39384-1_29

17. Kibria, M. G., Murata, H. Convex Approximated Weighted Sum-Rate Maximization for Multicell Multiuser OFDM. IEICE Transactions on Fundamentals of Electronics Communications and Computer Sciences, 2017, 97(8), 1800-1805. https://doi.org/10.1587/transfun. E97.A.1800

18. Kihl, M., Bur, K., Mahanta, P., Coelingh, E. 3GPP LTE Downlink Scheduling Strategies in Vehicle-to -Infrastructure Communications for Traffic Safety Applications. In Proceedings IEEE Symposium on Computers and Communications, Cappadocia (ISCC 2012), Turkey, July 1-4, 2012, 448-453. https://doi.org/10.1109/ ISCC.2012.6249337

19. Ku, G., Walsh, M. L. Resource Allocation and Link Adaptation in LTE and LTE Advanced: A Tutorial. IEEE Communications Surveys and Tutorials, 2015, 17(3), 1605-1633. https://doi.org/10.1109/ COMST.2014.2383691

20. Lyu, X., Ni, W., Tian, H. Optimal Schedule of Mobile Edge Computing for Internet of Things Using Partial Information. IEEE Journal on Selected Areas in Communications, 2017, 35(11), 2606-2615. https://doi. org/10.1109/JSAC.2017.2760186

21. Martynyuk, A. On the Theory of the Direct Method of A. M. Lyapunov. Doklady Akademii Nauk, 2006, (3), 309312. https://doi.org/10.1134/S1064562406030161

22. Mazzola, M., Schaaf, G., Stamm, A. Safety-Critical Driver Assistance Over LTE: Toward Centralized ACC. IEEE Transactions on Vehicular Technology, 2016, 65(12), 9471-9478. https://doi.org/10.1109/ TVT.2016.2617320

23. Neely, M. J. Stochastic Network Optimization with Application to Communication and Queueing Systems. Synthesis Lectures on Communication Networks. Morgan \& Claypool, 2010. https://doi.org/10.2200/ S00271ED1V01Y201006CNT007

24. Pereira Coutinho, W., Do Nascimento, R. Q., Alves Pessoa, A., Subramanian, A. A Branch-and-Bound Algorithm for the Close-Enough Traveling Salesman Problem. INFORMS Journal on Computing, 2016, 28 (4), 752-765. https://doi.org/10.1287/ijoc.2016.0711

25. Rupp, M., Schwarz, S., Taranetz, M. Signals and Communication Technology. The Vienna LTE-Advanced Simulators. Springer, 2016. https://doi.org/10.1007/978-98110-0617-3 
26. Schwarz, S., Ikuno, J. C., Šimko, M. Pushing the Limits of LTE: A Survey on Research Enhancing the Standard. IEEE Access, 2013, 1(4), 51-62. https://doi.org/10.1109/ ACCESS.2013.2260371

27. Shevitz, D., Paden, B. Lyapunov Stability Theory of Nonsmooth Systems. IEEE Transactions on Automatic Control, 1994, 39(9), 1910-1914. https://doi. org/10.1109/9.317122

28. Tung, L. C., Gerla, M. LTE Resource Scheduling for Vehicular Safety Applications. 2013 10th Annual Conference on Wireless On-demand Network Systems and Services (WONS), Banff, Canada, May 18-20, 2013, 116118. https://doi.org/10.1109/WONS.2013.6578333

29. Venčkauskas, A., Morkevicius, N., Jukavičius, V., Damaševičius, R., Toldinas, J., Grigaliūnas, Š. An Edge-Fog Secure Self-Authenticable Data Transfer Protocol. Sen- sors, 2019, 19(16), 3612-3623. https://doi.org/10.3390/ s19163612

30. Weeraddana, P. C., Codreanu, M., Latva-aho, M., Ephremides, A. Weighted Sum-Rate Maximization for a Set of Interfering Links via Branch and Bound. IEEE Transactions on Signal Processing, 2011, 59(8), 3977-3996. https://doi.org/10.1109/TSP.2011.2152397

31. Wei, Q., Wang, L., Feng, Z. Wireless Resource Management in LTE-U Driven Heterogeneous V2X Communication Networks. IEEE Transactions on Vehicular Technology, 2018, 67(8), 7508-7522. https://doi. org/10.1109/TVT.2018.2823313

32. Zheng, K., Zheng, Q., Chatzimisios, P., Xiang, W., Zhou, Y. Heterogeneous Vehicular Networking: A Survey on Architecture, Challenges, and Solutions. IEEE Communication Survey and Tutorials, 2015, 17(4), 2377-2396. https://doi.org/10.1109/COMST.2015.2440103 